\title{
The Effectiveness of a Pseudo Inverse Extended Born Operator to Handle Lateral Heterogeneity for Imaging and Velocity Analysis Applications
}

\author{
Abdullah Alali (abdullah.alali.1@kaust.edu.sa) \\ Bingbing Sun (bingbing.sun@kaust.edu.sa) \\ Tariq Alkhakifah (tariq.alkhalifah@kaust.edu.sa) \\ King Abdullah University of Science and Technology (KAUST), \\ Physical Sciences and Engineering Division, \\ Thuwal, Saudi Arabia, 23955.
}


(February 1, 2020)

Running head: Pseudo Inverse Extended Born Operator

\begin{abstract}
Wave equation based migration velocity analysis (WEMVA) techniques aim to construct a kinematically accurate velocity model for imaging or as an initial model for full waveform inversion (FWI) applications. The most popular WEMVA method is differential semblance optimization (DSO), where the velocity model is iteratively updated by minimizing the unfocused energy in an extended image volume. However, DSO suffers from artifacts, courtesy of the adjoint operator used in imaging, leading to poor convergence. Recent findings show that true amplitude imaging plays a significant role in enhancing the DSO's gradient and reducing the artifacts. Here, we focus on a pseudo inverse operator to the horizontally extended Born as a true amplitude imaging operator. For laterally inhomogenuous models, the operator required a derivative with respect to a vertical shift. Extending the image vertically to evaluate such a derivative is costly and impractical. The inverse operator can be simplified in laterally homogeneous models. We derive an extension of the approach to apply the full inverse formula and evaluate the derivative efficiently. We simplified the implementation by applying the derivative to the imaging condition and utilize the relationship between the source and receiver wavefields and the vertical shift. Specifically, we verify the effectiveness of the approach using the Marmousi model and show that the term required for the lateral inhomogeneity treatment has a relatively small impact on the results for many cases. We then apply the operator in DSO and invert for an accurate macro-velocity model, which can serve as an initial velocity model for FWI.
\end{abstract}

Keywords: Velocity Analysis, MVA, Imaging, FWI. 


\section{INTRODUCTION}

An accurate macro-velocity model is essential in prestack depth migration to obtain the correct image and in full waveform inversion (FWI) (Lailly 1983; Tarantola 1984) to avoid the cycle skipping for convergence (Alkhalifah 2016; Sun and Alkhalifah 2019). Conventional velocity model building tools are based on traveltime or ray tracing methods, which utilize the high-frequency asymptotic approximation and fail to invert for an accurate velocity in complex regions where multi-arrival and shadow zone occur (Fei et al. 1996). On the contrary, wave equation methods using the full waveform information without approximations can lead to a more robust velocity tomography method.

Wave Equation Migration Velocity Analysis (WEMVA) techniques are developed to determine an accurate background velocity model using the wave equation. The traditional application of MVA involves splitting the data into subsets of common shot or common offset gathers and then migrate them separately (Symes 2008). The image should be independent of the acquisition geometry, which means any inconsistency in the common image gathers (CIGs) and the resulting residuals are due to the inaccurate model used in migration. This type of MVA is sensitive to kinematic artifacts when the model is complex (Symes 2008). For more robust MVA methods, we need to migrate the full data sets and to obtain the subsurface offsets (Rickett and Sava 2002), time shifts (Sava and Fomel 2006) or angle gathers (Sava and Fomel 2003). By measuring the unfocusing of the subsurface offset and the time shift gathers, or the unflatteness of the angle gathers, we can correct the velocity model accordingly (Symes 2008; Kalita and Alkhalifah 2016; Chauris and Cocher 2017; Sun and Alkhalifah 2017).

A critical aspect in MVA is how to design a misfit function in the subsurface domain to measure the inaccuracy of the velocity model. The most popular method is differential semblance optimization (DSO) introduced by Symes (2008). DSO is implemented by applying a spatial or temporal extension to the image domain first (Sava and Fomel 2006; Rickett and Sava 2002; Symes 2008) and then penalizing the energy residual in this nonphysical extension. In this paper, we will only consider the horizontal space shift known as the subsurface offset. The main reason for increasing the dimensions of the image is to be able to match the dimensions of the data even with an incorrect velocity. The velocity is considered to be accurate when the energy is focused around the zero-subsurface offset, lower (higher) velocity will form a frown (smile) shape in the CIGs. Another way to assess the quality of the velocity is the flatness of events in the scattering angle domain, which can be obtained by a slant-stack transform of the subsurface offset gather (Sava and Fomel 2003).

Although DSO shows successful results in some studies (Symes 2008; Shen 2005; Mulder and ten Kroode 2002), it has some limitations. According to Fei and Williamson (2010), the gradient of DSO suffers from artifacts and undesired oscillations. Also, for Reverse Time Migration (RTM) (Baysal, Kosloff and Sherwood 1983), the reflected waves generate unwanted artifacts in the image, which affect the DSO performance (Liu, Symes and Li 2014). Moreover, the CIGs in the extended domain are affected by the uneven illumination due to limited acquisition geometry (Dafni and Symes 2016). All these factors affect the optimization process and reduce its chances for a stable convergence (Symes 2008; Sun and Alkhalifah 2017; Alkhalifah and Wu 2017).

Many studies have addressed those issues: Fei and Williamson (2010) reduced DSO 
artifacts by introducing a 90-degree phase shift to the sub-surface offset gathers by applying a differential operator to the objective function; Lameloise, Chauris and Noble (2015) improved the gradient efficiency by using quantitative migration; and most recent studies by Chauris and Cocher (2017) and Hou and Symes (2018) proposed to obtain the extended images using an approximate inverse rather than adjoint to the extended Born. This type of velocity analysis is called Inversion Velocity Analysis (IVA) instead of MVA to highlight that an inverse operator is used for imaging instead of the adjoint (Chauris and Cocher 2017; Hou and Symes 2018). The approximate inverse preserves the true amplitude and reduces the artifacts noise even when an incorrect velocity is used. Different formulas were derived for the approximate inverse (ten Kroode 2012; Hou and Symes 2015; Chauris and Cocher 2017; Hou and Symes 2018). They all take into account the amplitude terms (geometrical spreading), deconvolution of the seismic source, and the uneven illumination of the subsurface (Chauris and Cocher 2018).

In this paper, we will focus on the pseudo inverse operator developed by Chauris and Cocher (2017). The operator was developed based on the horizontal extension, but it requires a derivative of the image with respect to a vertical extension in laterally variant models. Implementing the vertical extension to take its derivative is costly, and not practical to implement in many cases. Thus, Chauris and Cocher (2017) approximated the inverse operator and neglected this derivative. Under this approximation, the operator provides good results for laterally variant models. In this paper, we will provide an explicit justification for the approximation.

The main objective of this work is to compute the derivative without applying the vertical extension and implement the full pseudo inverse operator in laterally variant models. This is accomplished by taking the derivative to the extended imaging condition evaluated at zero subsurface offset position. We also want to emphasize the robustness of the IVA in providing an accurate macro model that can be used in imaging or as a non cycle-skipped initial model in FWI.

The paper is organized as follows: We start first by reviewing the theory of imaging using the adjoint of the Born operator and its inverse. Then, we show how to implement the full pseudo inverse formula by computing the derivative of the vertical extension. Numerical evidence, shown later in this paper, suggests that the new term has a minor influence on the inversion even in heterogeneous models. This finding justifies the approximation of the pseudo inverse operator and that the vertical extension derivative can be safely neglected. At last, we present examples to demonstrate the effectiveness of the pseudo inverse operator in imaging and IVA in obtaining a good initial model for FWI.

\section{THEORY}

In this section, we first revisit the concept of Born operators, and their pseudo inverse, and then we introduce an efficient way to calculate the full pseudo inverse operator for laterally varying background media. Finally, we will formulate the optimization problem for IVA. 


\section{Born Operators}

Extended Born modelling maps the extended reflectively image $I(\mathbf{x}, h)=\frac{2 \delta v(\mathbf{x}, h)}{v^{3}(\mathbf{x})}$, where $v$ is the velocity and $\delta v$ is the velocity perturbation, into seismic data (Symes 2008). The extended Born forward modelling operator is given by

$$
\begin{aligned}
d\left(\mathbf{x}_{r} ; \mathbf{x}_{s}, \omega\right)= & -(i \omega)^{2} \Omega(\omega) \\
& \int G\left(\mathbf{x}_{s}, \mathbf{x}-h, \omega\right) I(\mathbf{x}, h) G\left(\mathbf{x}+h, \mathbf{x}_{r}, \omega\right) d h d \mathbf{x}
\end{aligned}
$$

where $\mathbf{x}$ is the spacial coordinates in $2 \mathrm{D}(\mathrm{x}, \mathrm{z})$ and $\omega$ is the frequency. $\Omega$ is the source term, for example a Ricker wavelet. The two Green's functions $G\left(\mathbf{x}_{s}, \mathbf{x}+h, \omega\right)$ and $G(\mathbf{x}-$ $\left.h, \mathbf{x}_{r}, \omega\right)$ satisfy the wave equation for a source located at $\mathbf{x}_{s}$ and receivers positioned at $\mathbf{x}_{r}$, respectively. $h$ is a horizontal extension and represents the subsurface half-offset. To reconstruct the image, one can use the adjoint operator of equation 1 as,

$$
\begin{aligned}
I_{a d j}(\mathbf{x}, h)= & -\int(i \omega)^{2} \Omega^{*}(\omega) G^{*}\left(\mathbf{x}_{s}, \mathbf{x}-h, \omega\right) \\
& d\left(\mathbf{x}_{r} ; \mathbf{x}_{s}, \omega\right) G^{*}\left(\mathbf{x}+h, \mathbf{x}_{r}, \omega\right) d \omega d \mathbf{x}_{s} d \mathbf{x}_{r} .
\end{aligned}
$$

The adjoint is similar to RTM, where the image is obtained by a cross-correlation between the incident source and the back-propagated receiver wavefields. The adjoint degrades the amplitude and contains low frequency artifacts. To achieve true amplitude imaging and reduce the artifacts, an inverse operator rather than the adjoint is used (ten Kroode 2012; Hou and Symes 2015; Chauris and Cocher 2017). Under the high frequency assumption, an approximate inverse to the extended Born was derived by Chauris and Cocher (2017) that reads,

$$
\begin{aligned}
I_{i n v}(\mathbf{x}, h)= & \frac{32}{v_{0+} v_{0-}} D_{p} \int \frac{\tilde{\Omega}(\omega)}{i \omega} D_{s_{z}} G^{*}\left(\mathbf{x}_{s}, \mathbf{x}-h, \omega\right) \\
& d\left(\mathbf{x}_{r} ; \mathbf{x}_{s}, \omega\right) D_{r_{z}} G^{*}\left(\mathbf{x}+h, \mathbf{x}_{r}, \omega\right) d \omega d \mathbf{x}_{s} d \mathbf{x}_{r},
\end{aligned}
$$

with

$$
D_{p}=\frac{1}{2} \frac{1}{v_{0+} v_{0-}}\left(\left(v_{0+}^{2}+v_{0-}^{2}\right) D_{z}+\left(v_{0+}^{2}-v_{0-}^{2}\right) D_{h_{z}}\right)
$$

Here $v_{0+}=v(x+h)$ and $v_{0-}=v(x-h)$ are the shifted background velocities. $\tilde{\Omega}(\omega)$ is the inverse of $\Omega(\omega)$, which can be obtained as $\tilde{\Omega}=\frac{\Omega^{*}}{\|\Omega\|^{2}}$. $D_{r_{z}}$ and $D_{s_{z}}$ are derivatives with respect to the vertical dimension of the receiver and source positions, respectively. They are implemented by using a dipole source/receiver wavefield instead of a wavefield of a point source/receiver (Chauris and Cocher 2017). Figure 1 shows a sketch of the layout of the dipole source and receiver. The $D_{p}$ operator is a linear combination of two derivatives applied to the image, namely a vertical derivative $D_{z}$ and a derivative with respect to the vertical extension $D_{h_{z}}$ evaluated at zero.

[Figure 1 about here.]

Compared to the adjoint, the pseudo inverse has a first order integration of the inverted source instead of a second order derivative to the wavelet, as well as, vertical derivatives in front of the Green's function, which amount to applying cosines of take-off angles at the 
sources and receivers positions. Finally, it has the $D_{p}$ operator implemented to the image, which acts as multiplying the image with the cosines of half-opening angles and will be discussed in details in the following sections. The inverse operator also includes a scaling

factor $\frac{32}{v_{0+} v_{0-}}$ for the amplitude. More details about the derivation of these weights can be found in Chauris and Cocher (2017).

The $D_{p}$ operator in equation 4 contains two derivatives, which are $D_{z}$ taken at the image points and $D_{h_{z}}$ evaluated at $h_{z}=0$. Implementing the vertical extension $h_{z}$ to take its derivative is very costly and impractical to implement in many cases. Therefore, Chauris and Cocher (2017) approximate the formula and applied it without the $D_{h_{z}}$ term.

\section{Lateral Heterogeneity}

To compute the full inverse without applying the vertical extension, we propose to compute $D_{h_{z}}$ using the chain rule on the integrand of equation 3 which yields,

$$
\begin{aligned}
& \left.\frac{\partial}{\partial h_{z}} G^{*}\left(\mathbf{x}_{s}, x-h_{x}, z-h_{z}, \omega\right) G^{*}\left(x+h_{x}, z+h_{z}, \mathbf{x}_{r}, \omega\right)\right|_{h_{z}=0} \\
& =\int\left(\frac{\partial G^{*}\left(x+h_{x}, z, \mathbf{x}_{r}, \omega\right)}{\partial z} G^{*}\left(\mathbf{x}_{s}, x-h_{x}, z, \omega\right)\right. \\
& \left.-\frac{\partial G^{*}\left(\mathbf{x}_{s}, x-h_{x}, z, \omega\right)}{\partial z} G^{*}\left(x+h_{x}, z, \mathbf{x}_{r}, \omega\right)\right) d \omega .
\end{aligned}
$$

The vertical derivatives of the source and receiver wavefields are evaluated at the image points. The fact that $D_{h_{z}} I$ is evaluated at $h_{z}=0$ enables the evaluation of the derivative without applying any additional extension. For consistency, $D_{z}$ can be computed in a similar way using

$$
\begin{aligned}
& \frac{\partial}{\partial z} G^{*}\left(\mathbf{x}_{s}, x-h_{x}, z, \omega\right) G^{*}\left(x+h_{x}, z, \mathbf{x}_{r}, \omega\right) \\
& =\int\left(\frac{\partial G^{*}\left(x+h_{x}, z, \mathbf{x}_{r}, \omega\right)}{\partial z} G^{*}\left(\mathbf{x}_{s}, x-h_{x}, z, \omega\right)\right. \\
& \left.+\frac{\partial G^{*}\left(\mathbf{x}_{s}, x-h_{x}, z, \omega\right)}{\partial z} G^{*}\left(x+h_{x}, z, \mathbf{x}_{r}, \omega\right)\right) d \omega
\end{aligned}
$$

The vertical derivative of the source and receiver wavefields in the case of horizontal or nearly horizontal reflectors is almost the same. Hence $D_{z}$ in equation 6 is relatively large and $D_{h_{z}}$ in equation 5 is very small. On the contrary, if there are vertical reflectors, such as salt flanks, the image does not change with depth and in this case, $D_{z}$ would be extremely small and, applying a vertical shift would yield a change in the image and make $D_{h_{z}}$ relatively large. 


\section{Migration/Inversion Velocity Analysis}

In the subsurface-offset domain, the DSO objective function takes the form

$$
J(v)=\frac{1}{2}\|\mathbf{h} I(\mathbf{x}, h)\|^{2}=\frac{1}{2} \int h^{2} I^{2}(\mathbf{x}, h) d \mathbf{x} d h .
$$

The objective function is minimized by penalizing the energy residing in the nonphysical extension (i.e. away from the zero subsurface-offset $h=0$ ). The image $I$ in the objective function can be obtained by either the adjoint operator (equation 2) or by the pseudo inverse operator (equation 3) (Chauris and Cocher 2017; Hou and Symes 2018).

The gradient can be computed efficiently using the adjoint-state method (Plessix 2006). We consider $\mu_{s}, \mu_{r}$ and $\lambda_{0}$ as adjoint variables: $\mu_{s}$ is the adjoint-state source wavefield, $\mu_{r}$ is the adjoint-state receiver wavefield and $\lambda_{0}$ is the image residual. The gradient is derived in Appendix (A) and is given by the cross-correlation of the source wavefield $(S)$ and the receiver wavefield $(R)$ with their adjoint $\left(\mu_{s}\right)$ and $\left(\mu_{r}\right)$, respectively.

$$
\begin{aligned}
\nabla_{v} J= & -\int\left(i \omega^{2}\right) S\left(x_{s}, \mathbf{x}, \omega\right) \mu_{s}\left(x_{s}, \mathbf{x}, \omega\right) d x_{s} d \omega \\
& -\int\left(i \omega^{2}\right) R\left(x_{s}, \mathbf{x}, \omega\right) \mu_{r}\left(x_{s}, \mathbf{x}, \omega\right) d x_{s} d \omega
\end{aligned}
$$

The main difference between the gradient of MVA and IVA is the image residual $\lambda_{0}$, which is used to compute the adjoint-state wavefields. In MVA $\lambda_{0}$ is given by $\lambda_{0}=h^{2} I$ while in IVA it reads $\lambda_{0}=-\frac{32}{v^{2}} D_{z}^{2} h^{2} I$ provided the assumption $D_{p}=D_{z}$. To speed up the convergence, the conjugate gradient or the limited-memory Broyden-Fletcher-Goldfrab-Shanno (L-BFGS) algorithms could be applied.

\section{NUMERICAL EXAMPLE}

\section{Imaging Examples}

We will start with a simple two-layer model to demonstrate the features of the method. We follow this up with applying the approach on the challenging Marmousi model.

\section{A two layer model}

To show the effectiveness of the pseudo inverse operator in reducing the artifacts. We applied the approximate inverse and the adjoint operator on a two-layer model in three cases; (1) using a correct velocity model $\left(v_{c}=2.5 \mathrm{~km} / \mathrm{s}\right),(2)$ low velocity $\left(v_{l}=2.35 \mathrm{~km} / \mathrm{s}\right)$ and $(3)$ a high velocity $\left(v_{h}=2.65 \mathrm{~km} / \mathrm{s}\right)$. The reflector is located at $1 \mathrm{~km}$ depth. We synthetically employ 81 shots evenly spaced at $50 \mathrm{~m}$, and use a $15 \mathrm{~Hz}$ Ricker wavelet for the source. The data were recorded using 401 receivers placed every $10 \mathrm{~m}$ on the surface. Figure 2 shows images obtained from the adjoint operator (left column) and the inverse operator (right column) for the three velocity cases. For adjoint images, we can see that low frequency artifacts appear around the reflector as well as migration smiles. Whereas for the inverted images, the migration smiles and the majority of the artifacts are removed. In Figure 3, we show the CIG for the images in Figure 2. The first row represents the adjoint 
images and the second row is for the approximate inverse. The CIG for the pseudo inverse operator is clearly better than the one obtained from the adjoint operator since most of the artifacts are removed.

[Figure 2 about here.]

[Figure 3 about here.]

\section{The Marmousi Model}

To test the proposed approach for laterally inhomogeneous media and investigate the effect of the $D_{h_{z}}$ term relative to $D_{z}$ term in equation 4 , we apply the inverse operator on the Marmousi model given by Figure 4 . We used 180 shots separated by $50 \mathrm{~m}$ and used a 15 $\mathrm{Hz}$ Ricker wavelet for the source. The data were recorded using 903 receivers spaced $10 \mathrm{~m}$ apart. The formula is tested in two cases: 1) Using a smoothed model, given in Figure 5, of the Marmousi as a correct background velocity; 2) Using a lower background velocity by scaling down the smoothed version by a factor of 0.9 .

[Figure 4 about here.]

[Figure 5 about here.]

The comparison between the adjoint and the inverted images for the correct velocity case is given in Figure 6 and for the lower velocity case in Figure 7. In both figures, the image produced by the adjoint operator is in the top and the inverted image at the bottom. Beside the images are CIGs taken at $3.5 \mathrm{~km}$. As in the two-layer model, the inverted images have higher resolution and fewer artifacts than the adjoint images. This can be clearly seen from the CIGs. An important point is that when the velocity used in the migration is inaccurate (Figure 7), the CIG for the inverted image clearly has much less artifacts in the unfocused energy than the CIG for the adjoint. This will certainly improve the DSO gradient and lead to faster convergence.

[Figure 6 about here.]

[Figure 7 about here.]

According to the inverse formula (equation 3), the contribution of the $D_{h_{z}}$ term will be only in the non-physical images, namely the CIGs, and is expected to affect the areas with dipping reflectors. We show the $D_{h_{z}}$ image at $h_{x}=0$ in Figure 8 for the correct velocity. As expected, the image illuminates the dipping reflectors and the horizontal reflectors are very weak. However, not all the dipping reflectors are recovered, only those who have strong lateral variation in the model as suggested by equation 5 . To investigate the role of $D_{h_{z}}$ 
and its contribution to the full operator compared to $D_{z}$, we display the CIGs for both derivatives separately without multiplying them with the velocity functions, and then with multiplying them by the velocity functions (equation 4), in addition, we display the full $D_{p}$ operator, which are shown in Figure 9 for the correct velocity image and in Figure 10 for the low-velocity image. The CIG gathers are taken in four positions at $x=2 \mathrm{~km}$; where the layers are almost horizontal, $x=3 \mathrm{~km}$; where the layers are gently dipping, and also at $x=4.5 \mathrm{~km}$ and $6 \mathrm{~km}$; where the model has steep dips. Figure 9 shows good focusing at the zero offset for the $D_{z}$ part indicating that a correct velocity was used. On the contrary, the CIG for $D_{h_{s}}$ is mostly low energy noise and does not show any focusing except in very few points where the faults and the dipping reflectors are present in the model. For the low-velocity case (Figure 10), we see downward curvatures in all the panels in the CIG for $D_{z}$ courtesy of the low velocity. However, this is not obvious in the CIG for $D_{h_{z}}$ part and barely seen in the middle panels as in the correct velocity case.

[Figure 8 about here.]

[Figure 9 about here.]

[Figure 10 about here.]

\section{IVA Example}

We perform IVA on the Marmousi (II) model given in Figure 11(a), as it is usually a challenging example for velocity analysis. We synthetically deploy 282 sources spaced $60 \mathrm{~m}$ apart at $45 \mathrm{~m}$ depth with a $10 \mathrm{~Hz}$ Ricker wavelet. The data are recorded using 1132 receivers every $13 \mathrm{~m}$ at a $45 \mathrm{~m}$ depth. For the inversion, we used a linearly increasing 1D model given by Figure 11(b) as an initial model. We run the optimization for 50 iterations with a gradient descent method. The model converges after ten iterations as shown in Figure 12. Figure 11(c) shows the final result for the inversion, which represents the kinematics of the accurate model fairly well. To measure the quality of the inverted model, we use it as a background model for imaging and examine the subsurface-offset (ODCIG) and angle (ADCIG) gathers. The images are obtained using the pseudo inverse operator and are shown in Figure 13(a) for the initial model and Figure 13(b) for the inverted model. We can see that the reflectors are not in the correct positions in the initial image due to the velocity error but they appear in their true locations in the final image. Figure 14 shows eleven ODCIG panels for the images in Figure 13 with a maximum subsurface offset of 0.5 $\mathrm{km}$ from $x=0.75 \mathrm{~km}$ to $x=15.75 \mathrm{~km}$ spaced $1.5 \mathrm{~km}$ apart and Figure 15 shows the converted ADCIG with a maximum incident angle of 67.5 degrees. The final ODCIG and ADCIG show dramatic improvements in focusing and flatness, respectively, indicating that we have most likely converged to the right kinematic model.

[Figure 11 about here.] 
[Figure 12 about here.]

[Figure 13 about here.]

[Figure 14 about here.]

[Figure 15 about here.]

To further validate the final IVA model (Figure 11(c)), we use it as a starting model to perform FWI in which we minimize the L2-norm of the data misfit. The acquisition geometry is the same as in IVA with a Ricker wavelet of $7 \mathrm{~Hz}$ and a high-cut filter at $10 \mathrm{~Hz}$. We also implement FWI using the 1D linearly increasing velocity $V(z)$ as an initial model (Figure 11(b)). The final inversion results are given in Figure 16(a) for the $V(z)$ model and Figure 16(b) for the IVA model. For the former, we didn't converge to the correct model because the starting velocity is not sufficiently close to the true one. However, for the latter, most of the Marmousi structures are correctly recovered demonstrating the accuracy of the initial model given by IVA.

[Figure 16 about here.]

\section{DISCUSSION}

We derived an efficient method to compute the derivative with respect to the vertical extension without implementing the costly vertical shift. The cost of the proposed method in evaluating $D_{h_{z}}$ is twice the cost of the horizontally extended imaging. One can compute $D_{h_{z}}$ with higher cost by applying a central differences to an extended image in $h_{z}$ at $I\left(x, z, h_{x},+\Delta h z\right)$ and another image at $I\left(x, z, h_{x},-\Delta h z\right)$. In addition, our approach is based on the concept of the chain rule which is analytically more accurate than applying a central difference in which the accuracy depends on how small $\Delta h_{z}$ is.

By examining Figures 9(a), 9(b), 10(a) and 10(b), we notice that the $D_{z}$ CIG are higher in magnitude than the $D_{h_{z}}$ CIG. Moreover, after implementing the full terms and multiplying the proper velocity functions as in equation 4 (Figures $9(\mathrm{c}), 9(\mathrm{~d}), 10$ (c) and $10(\mathrm{~d}))$ the $D_{h_{z}}$ become even smaller in magnitude and equal to zero where $v_{0+}=v_{0-}$ and this can be seen clearly in the first panel that is taken at $x=2 \mathrm{~km}$. The combination of the two terms, which yields the full $D_{p}$ operator is given in Figures $9(\mathrm{e})$ and $10(\mathrm{e})$. We see that the $D_{p}$ is dominated by $D_{z}$ part and the term containing $D_{h_{z}}$ doesn't show any noticeable influence even in the dipping parts, which suggests that it can be ignored for this model. Since the effect of the $D_{h_{z}}$ term is relatively small, we ignored it in the IVA implementation.

The full $D_{p}$ derivative provides asymptotically an exact inverse. The difference between $D_{z}$ and $D_{h_{z}}$ in Figures 9 and 10, respectively, can be considered as the error of approximating $D_{p}$ to have only $D_{z}$. Figure 10 shows a larger difference, as compared to Figure 9 due to the greater spread of energy over h which results in higher error in the $D_{z}$. 
In the conventional DSO approach, the kinematics of the Marmousi model cannot be estimated due to the low frequency artifacts from imaging using the adjoint operator. The true-amplitude imaging and the elimination of the artifacts using the pseudo inverse formula enable DSO to converge even in a complex model such as the Marmousi. Although we only used the gradient descent method in the IVA, Figure 12 shows fast convergence which demonstrates the efficiency of the operator in velocity estimation.

Hou and Symes (2018) implemented IVA using a different inverse operator and estimated a good kinematic model. The approach in this paper is very similar. In fact, as mentioned in the introduction, all inverse formulas are asymptotically equivalent (Chauris and Cocher 2018). The main difference is that Hou and Symes (2018) applied the vertical sources and receivers derivatives on the observed data by taking advantage of the ghost in simple streamer geometry and shallow tow depth. Their implementation is restricted to the geometry of the streamer and might require modification in complicated geometry (Hou and Symes 2015). However, in equation 3, the derivatives are applied to the Green's functions and implementing the dipole is simpler in this case.

The final image in Figure 13(b) and the FWI result in Figure 16(b) have some error in the deep part of the model especially in the middle region at the anticline between $x=8$ $\mathrm{km}$ and $x=10 \mathrm{~km}$. This is due to the high complexity of the model in this region. This suggests that even though we succeeded to recover most of the model accurately, there are still some challenges and area for improvements.

\section{CONCLUSION}

We utilized a pseudo inverse to the extended Born and showed its effectiveness in dealing with lateral inhomogeneity. The full pseudo inverse includes a derivative of the extended image with respect to a vertical shift, which can be costly. We derived a cost-effective evaluation of this derivative and demonstrate that the corresponding term has negligible influence on the inverse for reasonable models. Using the Marmousi model, we also showed that imaging using the pseudo inverse formula, rather than the adjoint, increases the resolution and reduces the artifacts in the extended image even if an inaccurate velocity is used. The pseudo inverse operator improves the efficiency of DSO and results in a good velocity estimation of the Marmousi in a few iterations. We showed also that the IVA velocity is a good starting model for FWI.

\section{ACKNOWLEDGMENT}

We thank the King Abdullah University of Science and Technology (KAUST) for its support and specifically the seismic wave analysis group (SWAG) members for their valuable insights. This work used the resources of the Supercomputing Laboratory at KAUST which we are grateful for. 


\section{APPENDIX A}

\section{GRADIENT CALCULATIOIN}

The DSO gradient is computed by the adjoint-state method (Plessix 2006). Using the extended Lagrangian formalism, the gradient is computed by extending the objective function (Equation 7) and finding the stationary points to all the state and adjoint state variables, all as independent variables. The gradient for MVA and IVA have similar forms and here we will focus on the derivation of IVA. Similar to Chauris and Cocher (2017) the new Lagrangian functional depends on $\left(v, \lambda, I, \lambda_{0}, I_{0}, \mu_{s}, S, \mu_{r}, R\right)$ and is defined as,

$$
\begin{aligned}
\tilde{J}= & \frac{1}{2}\|a I\|^{2}-<\lambda(\mathbf{x}, h), I(x, h)-D_{p} I_{0}(\mathbf{x}, h)> \\
& -<\lambda_{0}(\mathbf{x}, h), I_{0}-\int S^{*}\left(x_{s}, \mathbf{x}-h, \omega\right) R\left(x_{s}, \mathbf{x}+h, \omega\right) d x_{s} d \omega> \\
& -<\mu_{s}\left(x_{s}, \mathbf{x}, \omega\right), \mathcal{L} S-\Omega(\omega) \delta\left(x-x_{s}\right)> \\
& -<\mu_{r}\left(x_{s}, \mathbf{x}, \omega\right), \mathcal{L}^{*} R-\int d_{o b s}\left(x_{s}, \mathbf{x}, \omega\right) \delta\left(x-x_{r}\right) d r>
\end{aligned}
$$

where $<,>$ stands for the dot product. The first term is the original objective function and the other terms are, respectively, the definition of the image variable $I$ and $I_{0}$ associated with the adjoint variables $\lambda$ and $\lambda_{0}$, the definition of the source $S$ and receiver $R$ wavefields; solutions to the wave equation $\mathcal{L}$ with source term $\Omega$ and $d_{\text {obs }}$, and their adjoint variable $\mu_{s}$ and $\mu_{r}$. The total gradient reads,

$$
\begin{aligned}
\frac{\partial \tilde{J}}{\partial v}= & \frac{\partial J}{\partial v}+\frac{\partial \lambda}{\partial v} \frac{\partial \tilde{J}}{\partial \lambda}+\frac{\partial I}{\partial v} \frac{\partial \tilde{J}}{\partial I}+\frac{\partial \lambda_{0}}{\partial v} \frac{\partial \tilde{J}}{\partial \lambda_{0}}+\frac{\partial I_{0}}{\partial v} \frac{\partial \tilde{J}}{\partial I_{0}} \\
& +\frac{\partial \mu_{s}}{\partial v} \frac{\partial \tilde{J}}{\partial \mu_{s}}+\frac{\partial S}{\partial v} \frac{\partial \tilde{J}}{\partial S}+\frac{\partial \mu_{r}}{\partial v} \frac{\partial \tilde{J}}{\partial \mu_{r}}+\frac{\partial R}{\partial v} \frac{\partial \tilde{J}}{\partial R}
\end{aligned}
$$

By definition of the adjoint-staate variable, we set the derivative of $J$ with respect to the state variables $\left(\lambda, I, \lambda_{0}, I_{0}, \mu_{s}, S, \mu_{r}, R\right)$ to zero, yielding the following equations:

$$
\begin{gathered}
I(\mathbf{x}, h)=D_{p} I_{0}(\mathbf{x}, h), \\
I_{0}(\mathbf{x}, h)=\int S^{*}\left(x_{s}, h-x, \omega\right) R\left(x_{s}, h+x, \omega\right) d x_{s} d \omega,
\end{gathered}
$$

where $S$ is the forward propagated source and $R$ is the back propagated receiver wavefields, which are solutions to the wave equations,

$$
\begin{gathered}
\mathcal{L}^{*} R=\int d_{o b s}\left(x_{s}, \mathbf{x}, \omega\right) \delta\left(x-x_{r}\right) d r, \\
\mathcal{L} S=\Omega(\omega) \delta\left(x-x_{s}\right) .
\end{gathered}
$$

The derivatives of $J$ with respect to $S$ and $R$, set to 0 , give the expressions to calculate the adjoint source $\mu_{s}$ and the adjoint receiver $\mu_{r}$ : 


$$
\begin{aligned}
\mathcal{L}^{*} \mu_{s} & =\int \lambda_{0}(\mathbf{x}+h, h) R\left(x_{s}, \mathbf{x}+2 h, \omega\right) d h, \\
\mathcal{L} \mu_{r} & =\int \lambda_{0}(\mathbf{x}-h, h) S\left(x_{s}, \mathbf{x}-2 h, \omega\right) d h .
\end{aligned}
$$

The extended image residuals $\lambda$ and $\lambda_{0}$ is obtained using the derivative of $J$ with respect to $I$ and $I_{0}$ :

$$
\begin{array}{rlrl}
\lambda & \lambda=a^{2} I, \\
\lambda_{0} & =D_{p}^{*} \lambda=D_{p}^{*} a^{2} D_{p} I_{0}, \\
& =-\frac{32}{v^{2}} a^{2} D_{z}^{2} I_{0} & \text { (After simplifying } \left.D_{p} \text { to } D_{z}\right), \\
& =a^{2} I_{0} & \text { (For the adjoint version). }
\end{array}
$$

The final expression for the gradient is given in equation 7 . 


\section{LIST OF FIGURES}

1 Dipole source and receiver wavefields, the red line indicates the negative pole while the green line represents the positive pole.

2 Images using the adjoint operator for the two-layer model in case of (a) low velocity, (c) correct velocity and (e) high velocity, and using the inverse operator for the same cases in (b), (d) and $(\mathrm{g})$, respectively.

3 CIG of the images in Figure 2 taken at $\mathrm{x}=2 \mathrm{~km}$. (a), (b) and (c) for the adjoint operator and (d), (e) and (f) for the inverse operator both corresponding to low, correct and high velocity, respectively.

4 Marmousi model

5 Smooth Marmousi

6 (a) adjoint and (b) inverted image for the smoothed velocity with a CIG taken at $\mathrm{x}=3.5 \mathrm{~km}$.

7 (a) adjoint and (b) inverted image for the low velocity by a factor of 0.9 with a CIG taken at $\mathrm{x}=3.5 \mathrm{~km}$.

$8 D_{h_{z}}$ applied to the image at $h_{x}=0$.

9 CIGs for the correct velocity taken at $x=2,3,4.5$ and $6 \mathrm{~km}$ for (a) $D_{z}$ and (b) $D_{h_{z}}$ applied to the images and after multiplying them with the proper velocity weights in (c) and (d), respectively. (e) is the full $D_{p}$ operator.

10 CIGs for the low velocity taken at $x=2,3,4.5$ and $6 \mathrm{~km}$ for (a) $D_{z}$ and (b) $D_{h_{z}}$ applied to the images and after multiplying them with the proper velocity weights in (c) and (d), respectively. (e) is the full $D_{p}$ operator.

11 (a) is Marmousi (II) true model, (b) is the initial model and (c) the inverted model

12 The convergence curve of the normalized objective function.

13 (a) is initial image and (b) the final image.

14 (a) is initial ODCIG and (b) is the final ODCIG. They are computed from $\mathrm{x}=0.75$ $\mathrm{km}$ to $\mathrm{x}=15.75 \mathrm{~km}$ spaced by $1.5 \mathrm{~km}$ with maximum offset of $0.5 \mathrm{~km}$

15 (a) is initial ADCIG and (b) is the final ADCIG obtained by converting the gathers in Figure 14 with a maximum incident angle of 67.5 degree.

16 FWI results staring from the initial models (a) linearly increasing $\mathrm{v}(\mathrm{z})$ model given in Figure 11(b), and (b) the IVA result shown in Figure 11(c). 


\section{REFERENCES}

Alkhalifah T. 2016. Full-model wavenumber inversion: An emphasis on the appropriate wavenumber continuation. Geophysics 81, (3), R89-R98.

Alkhalifah T. and Wu Z. 2017. Migration velocity analysis using pre-stack wave fields. Geophysical Prospecting 65, (3), 639-649.

Baysal E., Kosloff D. D. and Sherwood J. W. C. 1983. Reverse time migration. Geophysics 48, (11), 1514-1524.

Chauris H. and Cocher E. 2017. From migration to inversion velocity analysis. Geophysics 82, (3), S207-S223.

Chauris H. and Cocher E. 2018. Review of different expressions for the extended Born approximate inverse operator. 80th EAGE Annual Meeting, Copenhagen, Denmark, Extended Abstracts, Workshop Programme WS07.

Dafni R. and Symes W. W. 2016. Kinematic artifacts in the subsurface-offset extended image and their elimination by a dip-domain specularity filter. Geophysics 81, (6), S477S495.

Fei T., Fehler C., Hildebrand S. T. and Chilcoat S. R. 1996. Depth migration artifacts associated with first-arrival traveltime. 66th SEG Annual Meeting, Denver, Colorado, USA, Expanded Abstracts, 499-502.

Fei W. and Williamson P. 2010. On the gradient artifacts in migration velocity analysis based on differential semblance optimization. 80th SEG Annual Meeting, Denver, Colorado, USA, Expanded Abstracts, 4071-4076.

Hou J. and Symes W. W. 2015. An approximate inverse to the extended Born modeling operator. Geophysics 80, (6), R331-R349.

Hou J. and Symes W. W. 2018. Inversion velocity analysis in the subsurface-offset domain. Geophysics 83, (2), R189-R200.

Kalita M. and Alkhalifah T. 2016. Common-image gathers using the excitation amplitude imaging condition. Geophysics 81, (4), S261-S269.

Lailly P. 1983. The seismic inverse problem as a sequence of before stack migrations. Conference on inverse scattering: theory and application, pp. 206-220. Siam Philadelphia, PA.

Lameloise C.-A., Chauris H. and Noble M. 2015. Improving the gradient of the imagedomain objective function using quantitative migration for a more robust migration velocity analysis. Geophysical prospecting 63, (2), 391-404.

Liu Y., Symes W. and Li Z. 2014. Inversion velocity analysis via differential semblance optimization. 76th EAGE Annual Meeting, Amsterdam, The Netherlands, Extended Abstracts, Th P02 07.

Mulder W. and ten Kroode A. 2002. Automatic velocity analysis by differential semblance optimization. Geophysics 67, (4), 1184-1191. 
Plessix R.-E. 2006. A review of the adjoint-state method for computing the gradient of a functional with geophysical applications. Geophysical Journal International 167, (2), 495-503.

Rickett J. E. and Sava P. C. 2002. Offset and angle-domain common image-point gathers for shot-profile migration. Geophysics 67, (3), 883-889.

Sava P. and Fomel S. 2006. Time-shift imaging condition in seismic migration. Geophysics 71, (6), S209-S217.

Sava P. C. and Fomel S. 2003. Angle-domain common-image gathers by wavefield continuation methods. Geophysics 68, (3), 1065-1074.

Shen P. 2005. Wave equation migration velocity analysis by differential semblance optimization. Ph. D. thesis, Rice University.

Sun B. and Alkhalifah T. 2017. Automatic Wave Equation Migration Velocity Analysis by Focusing Subsurface Virtual Sources. Geophysics 83, (2), U1-U8.

Sun B. and Alkhalifah T. 2019. The application of an optimal transport to a preconditioned data matching function for robust waveform inversion. Geophysics 84, no. 6, R923-R945.

Symes W. W. 2008. Migration velocity analysis and waveform inversion. Geophysical Prospecting 56, (6), 765-790.

Tarantola A. 1984. Inversion of seismic reflection data in the acoustic approximation. Geophysics 49, (8), 1259-1266.

ten Kroode F. 2012. A wave-equation-based Kirchhoff operator. Inverse Problems 28, (11), 115013. 


\section{LIST OF FIGURES}

1 Dipole source and receiver wavefields, the red line indicates the negative pole while the green line represents the positive pole. . . . . . . . . .

2 Images using the adjoint operator for the two-layer model in case of (a) low velocity, (c) correct velocity and (e) high velocity, and using the inverse operator for the same cases in (b), (d) and $(\mathrm{g})$, respectively. . . . . . . . .

3 CIG of the images in Figure 2 taken at $\mathrm{x}=2 \mathrm{~km}$. (a), (b) and (c) for the adjoint operator and (d), (e) and (f) for the inverse operator both corresponding to low, correct and high velocity, respectively. . . . . . . . . . . 20

4 Marmousi model . . . . . . . . . . . . . . . . . . 2

$5 \quad$ Smooth Marmousi . . . . . . . . . . . . . . . . . . 22

6 (a) adjoint and (b) inverted image for the smoothed velocity with a CIG taken at $\mathrm{x}=3.5 \mathrm{~km} \ldots \ldots \ldots \ldots \ldots$

7 (a) adjoint and (b) inverted image for the low velocity by a factor of 0.9 with a CIG taken at $\mathrm{x}=3.5 \mathrm{~km} \ldots \ldots \ldots . \ldots . \ldots 24$

$8 \quad D_{h_{z}}$ applied to the image at $h_{x}=0 \ldots \ldots \ldots \ldots \ldots$

$9 \quad$ CIGs for the correct velocity taken at $x=2,3,4.5$ and $6 \mathrm{~km}$ for (a) $D_{z}$ and (b) $D_{h_{z}}$ applied to the images and after multiplying them with the proper velocity weights in (c) and (d), respectively. (e) is the full $D_{p}$ operator. . .

10 CIGs for the low velocity taken at $x=2,3,4.5$ and $6 \mathrm{~km}$ for (a) $D_{z}$ and (b) $D_{h_{z}}$ applied to the images and after multiplying them with the proper velocity weights in (c) and (d), respectively. (e) is the full $D_{p}$ operator. . .

11 (a) is Marmousi (II) true model, (b) is the initial model and (c) the inverted model. . . . . . . . . . . . . . . . . . 28

12 The convergence curve of the normalized objective function. . . . . . . . . 29

13 (a) is initial image and (b) the final image. . . . . . . . . . . 30

14 (a) is initial ODCIG and (b) is the final ODCIG. They are computed from $\mathrm{x}=0.75 \mathrm{~km}$ to $\mathrm{x}=15.75 \mathrm{~km}$ spaced by $1.5 \mathrm{~km}$ with maximum offset of $0.5 \mathrm{~km}$

15 (a) is initial ADCIG and (b) is the final ADCIG obtained by converting the gathers in Figure 14 with a maximum incident angle of 67.5 degree. . . . . .

16 FWI results staring from the initial models (a) linearly increasing $v(z)$ model given in Figure 11(b), and (b) the IVA result shown in Figure 11(c) . . . . 


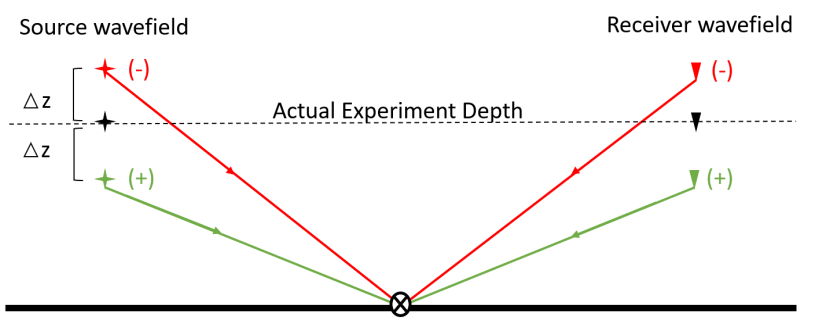

Figure 1: Dipole source and receiver wavefields, the red line indicates the negative pole while the green line represents the positive pole. 


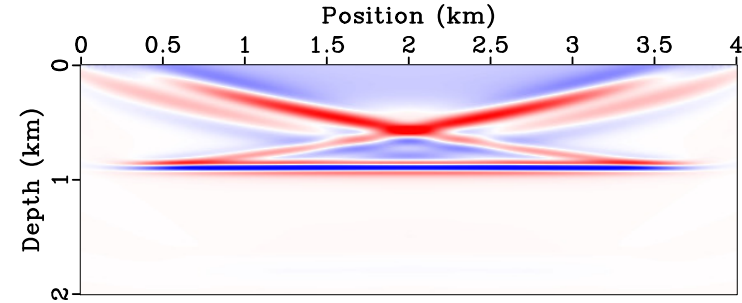

(a)

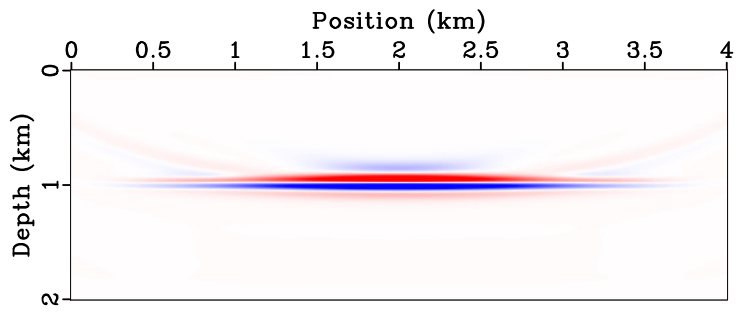

(c)

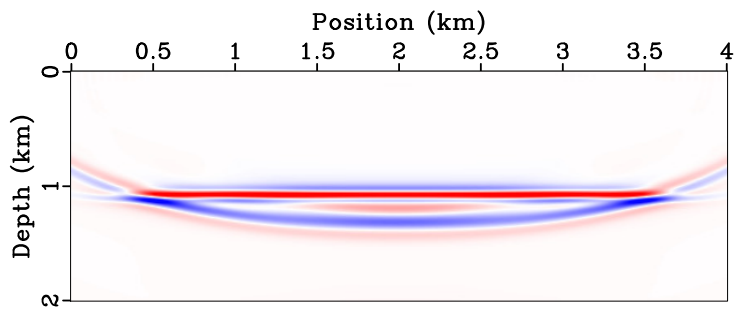

(e)

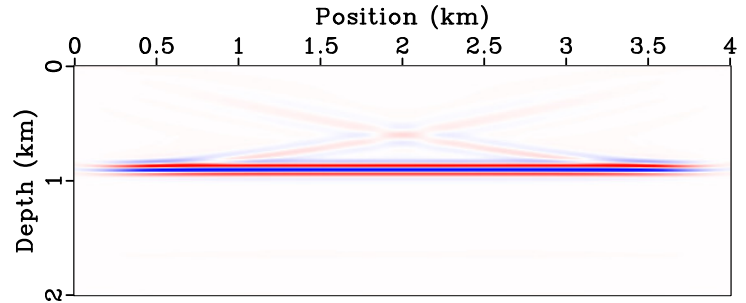

(b)

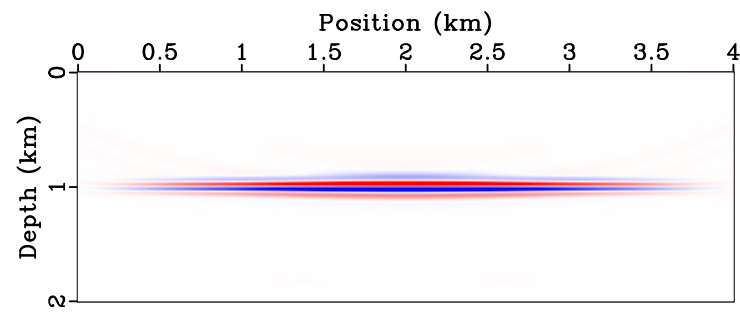

(d)

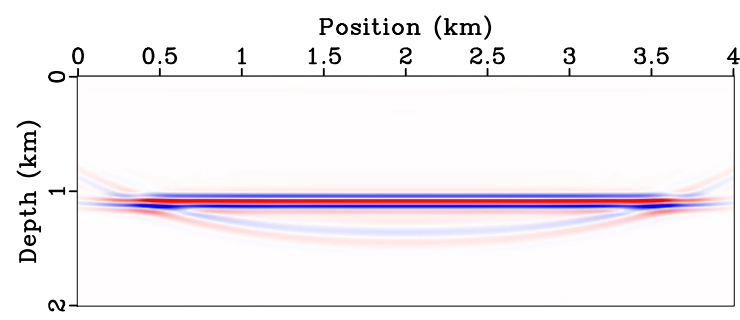

(f)

Figure 2: Images using the adjoint operator for the two-layer model in case of (a) low velocity, (c) correct velocity and (e) high velocity, and using the inverse operator for the same cases in (b), (d) and (g), respectively. 


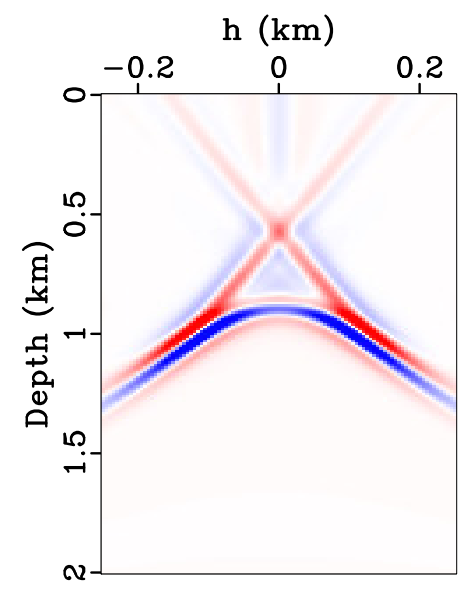

(a)

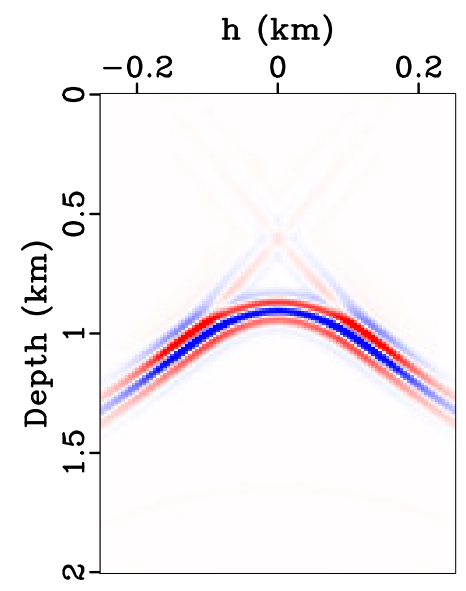

(d)

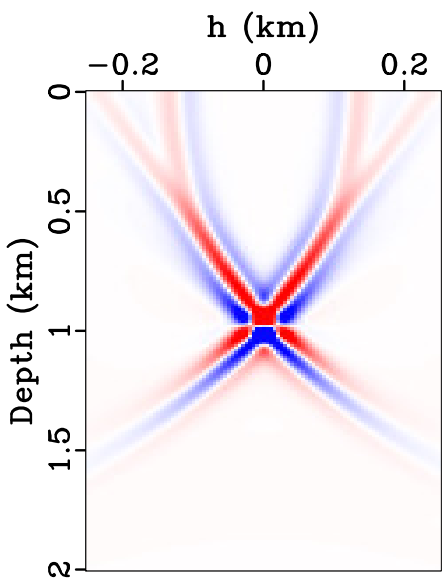

(b)

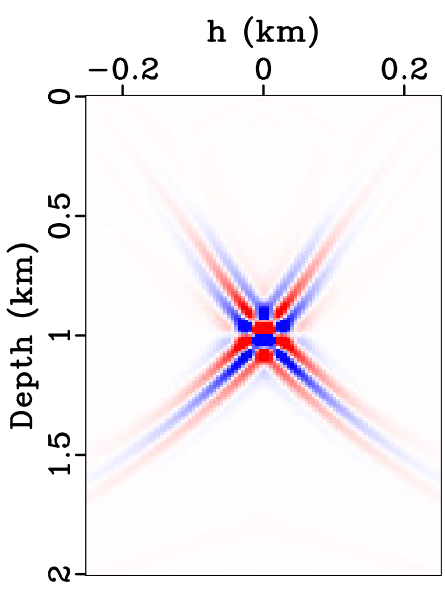

(e)

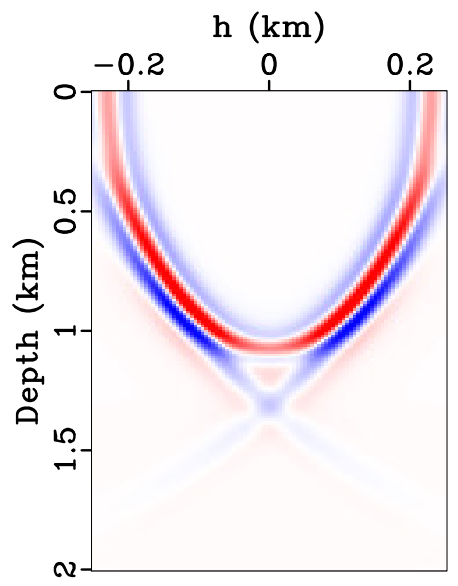

(c)

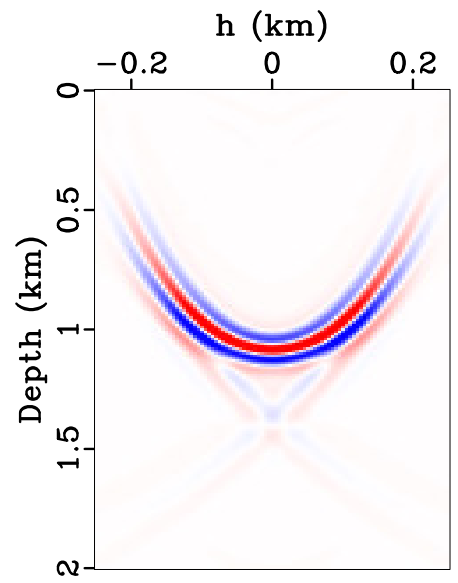

(f)

Figure 3: CIG of the images in Figure 2 taken at $\mathrm{x}=2 \mathrm{~km}$. (a), (b) and (c) for the adjoint operator and (d), (e) and (f) for the inverse operator both corresponding to low, correct and high velocity, respectively. 


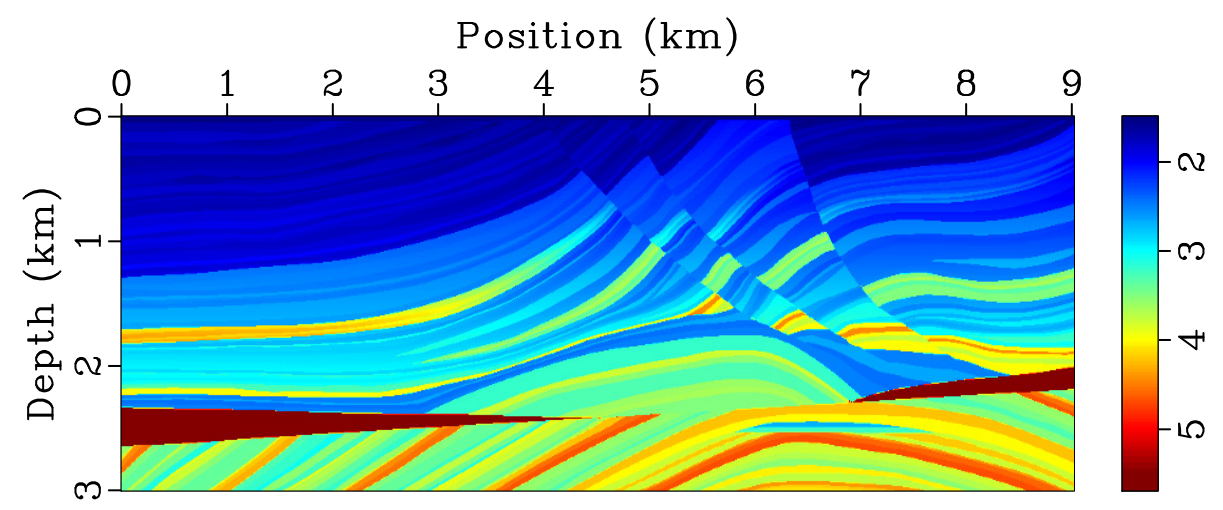

Figure 4: Marmousi model 


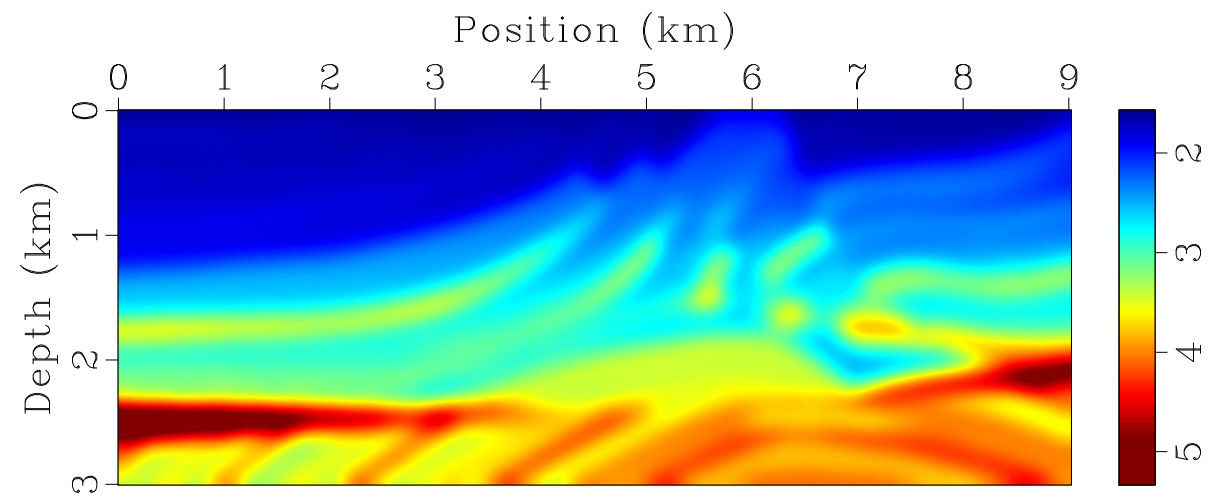

Figure 5: Smooth Marmousi 


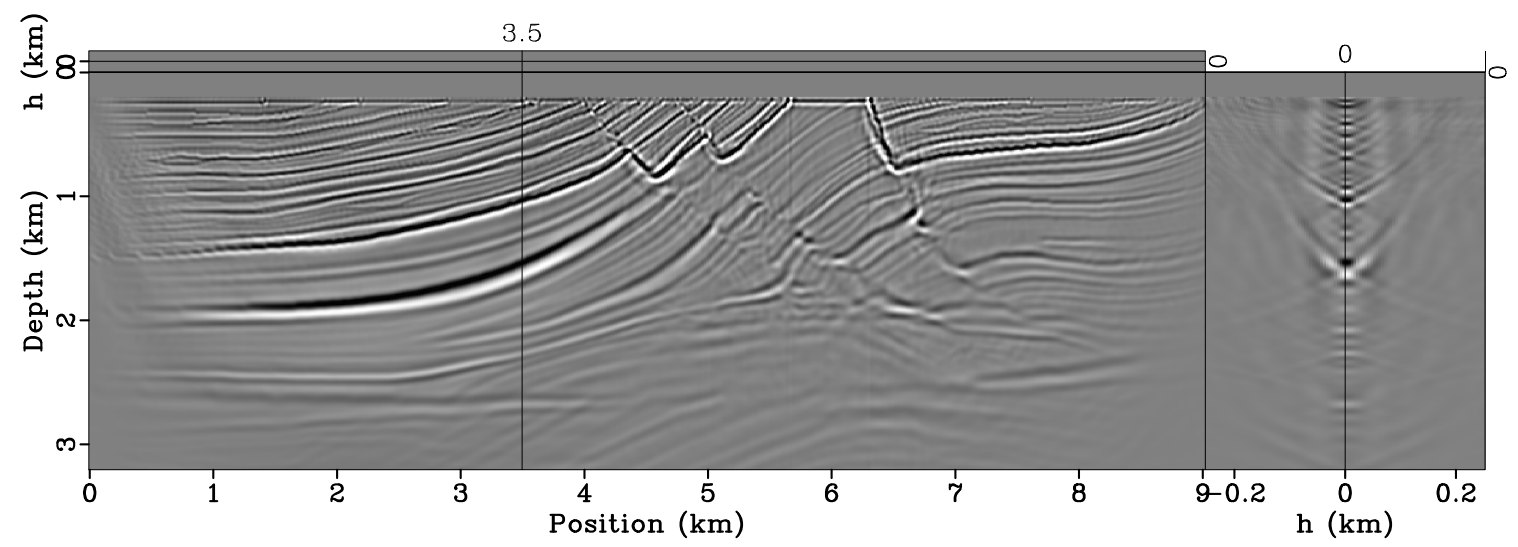

(a)

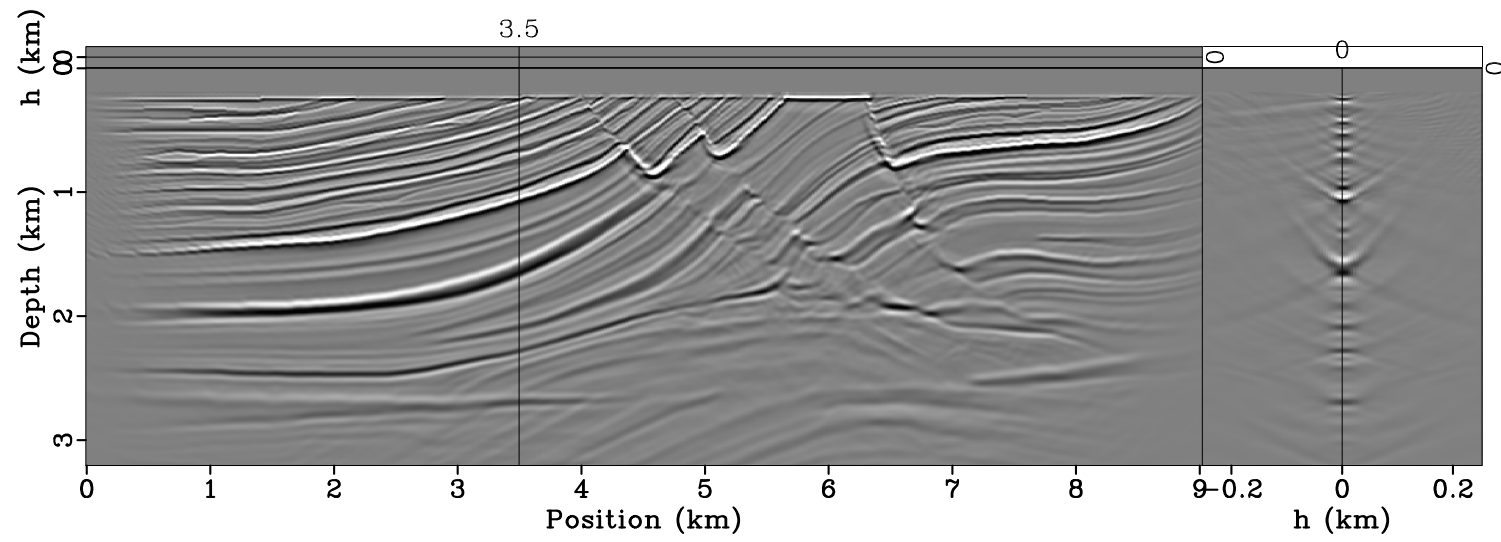

(b)

Figure 6: (a) adjoint and (b) inverted image for the smoothed velocity with a CIG taken at $\mathrm{x}=3.5 \mathrm{~km}$ 


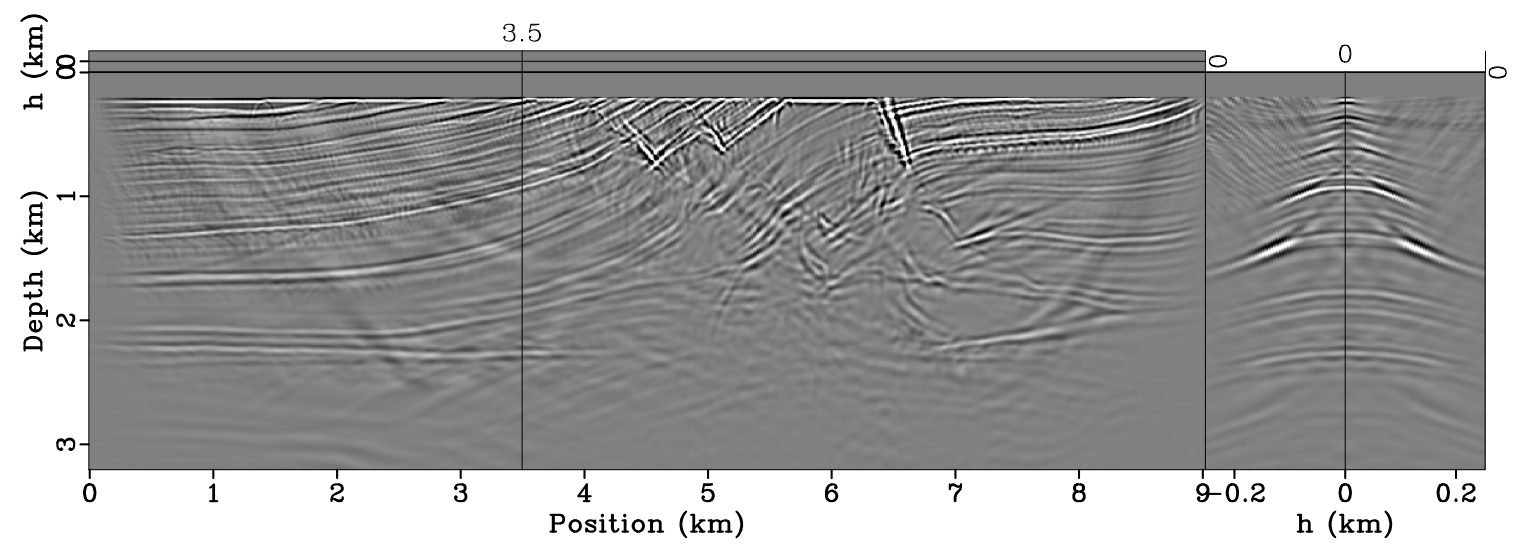

(a)

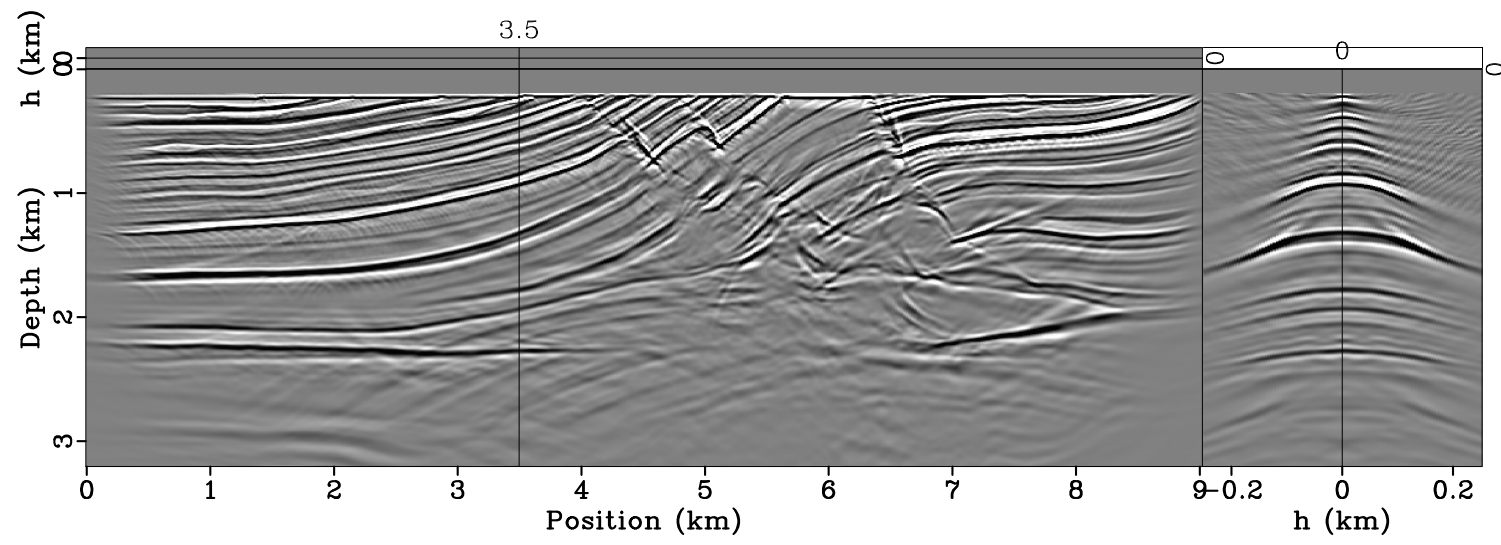

(b)

Figure 7: (a) adjoint and (b) inverted image for the low velocity by a factor of 0.9 with a CIG taken at $\mathrm{x}=3.5 \mathrm{~km}$ 


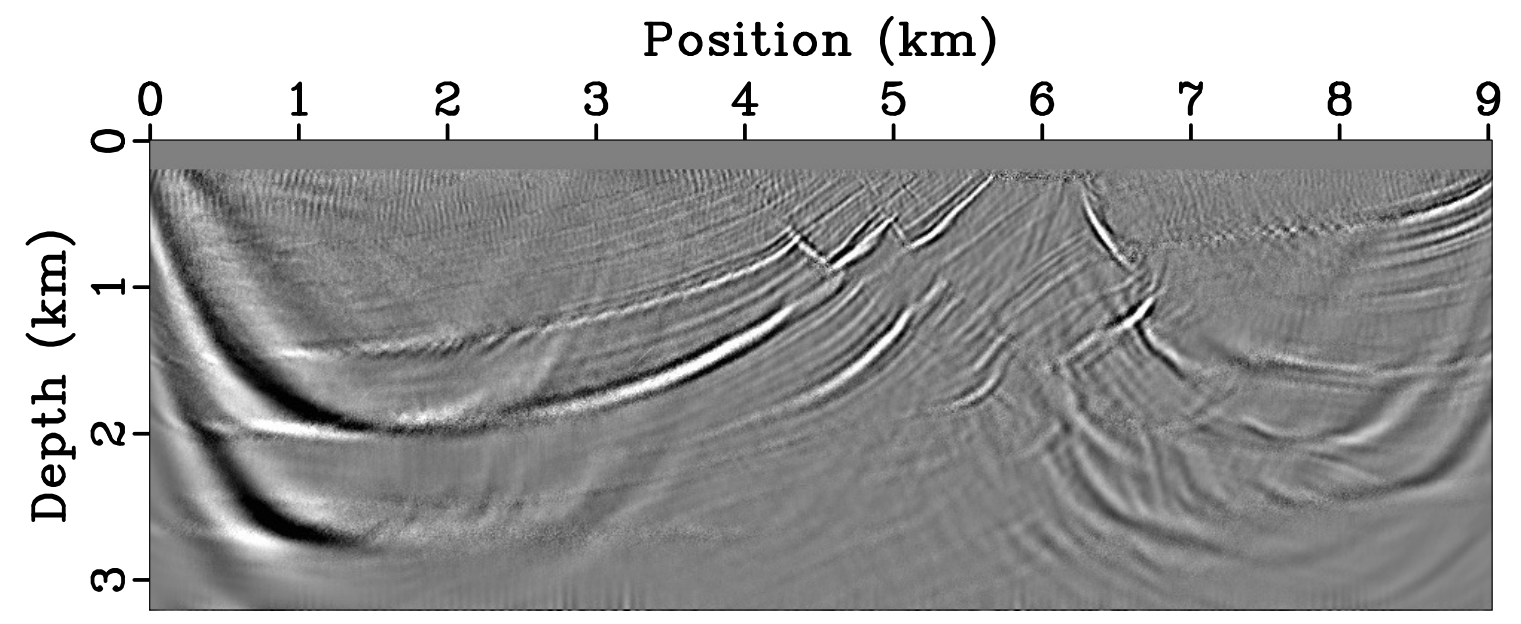

Figure 8: $D_{h_{z}}$ applied to the image at $h_{x}=0$. 


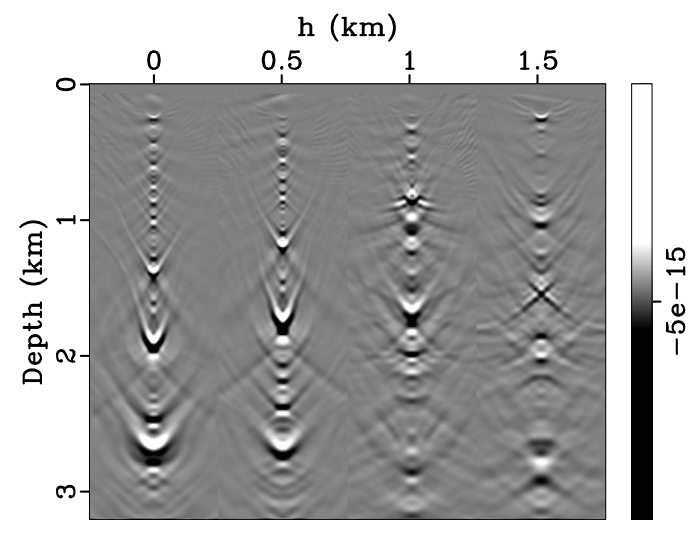

(a)

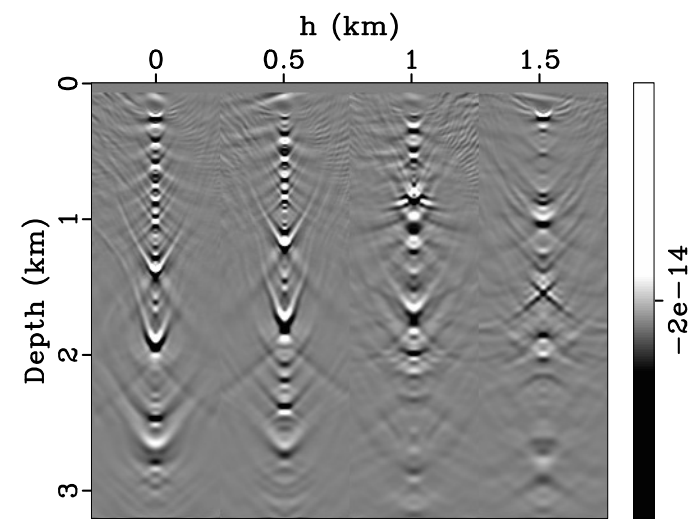

(c)

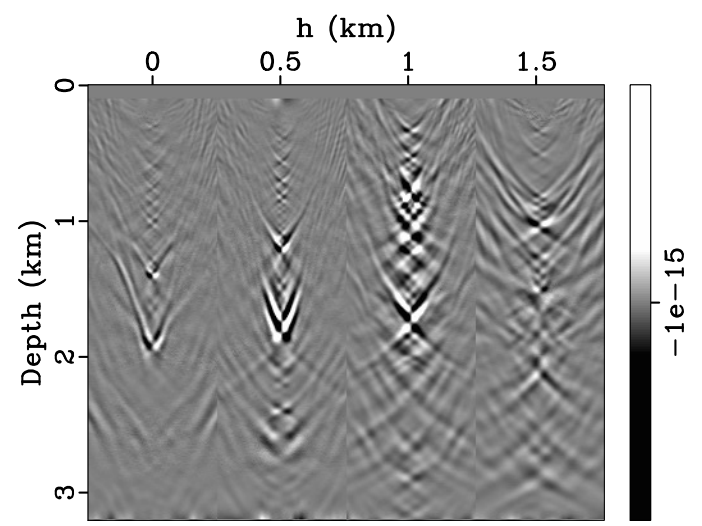

(b)

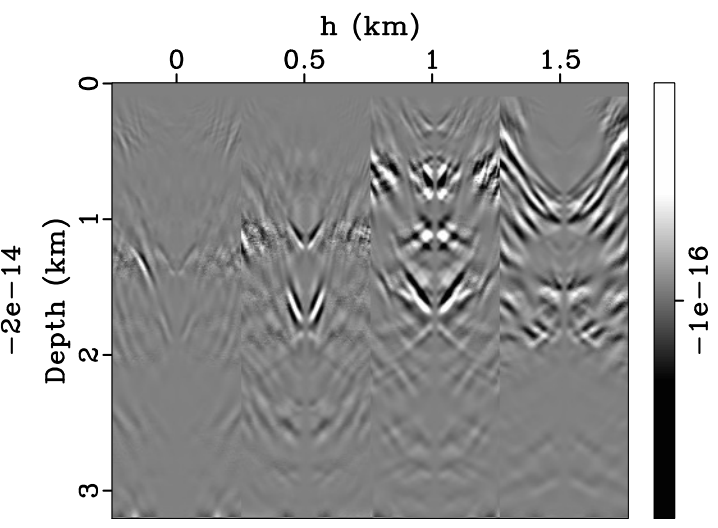

(d)

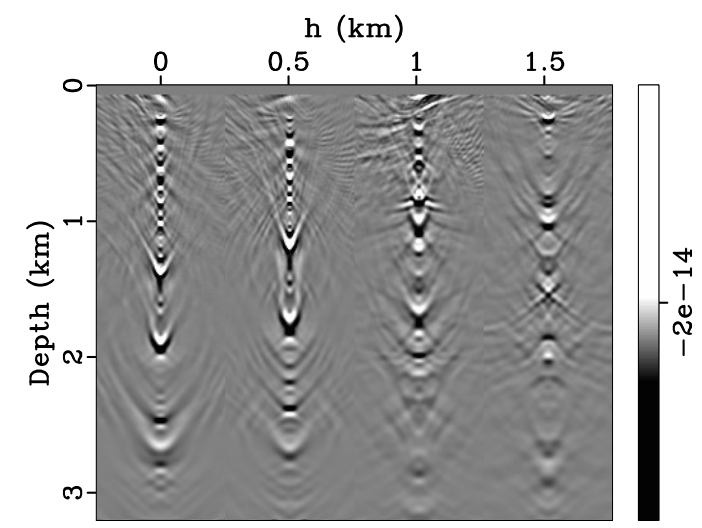

(e)

Figure 9: CIGs for the correct velocity taken at $x=2,3,4.5$ and $6 \mathrm{~km}$ for (a) $D_{z}$ and (b) $D_{h_{z}}$ applied to the images and after multiplying them with the proper velocity weights in (c) and (d), respectively. (e) is the full $D_{p}$ operator. 


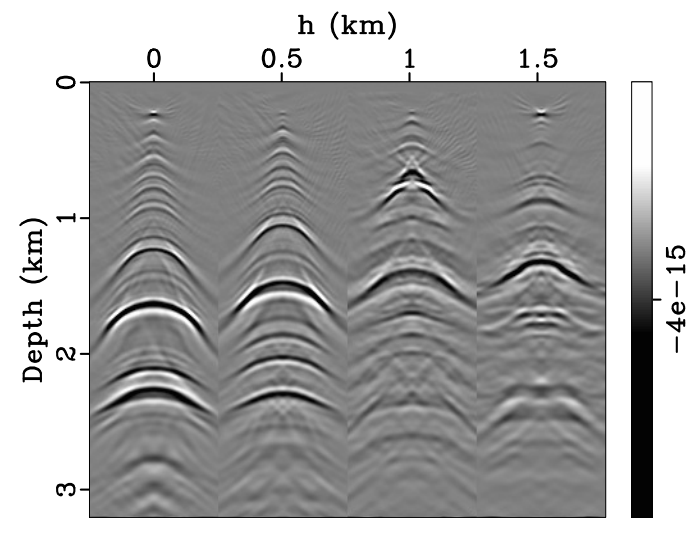

(a)

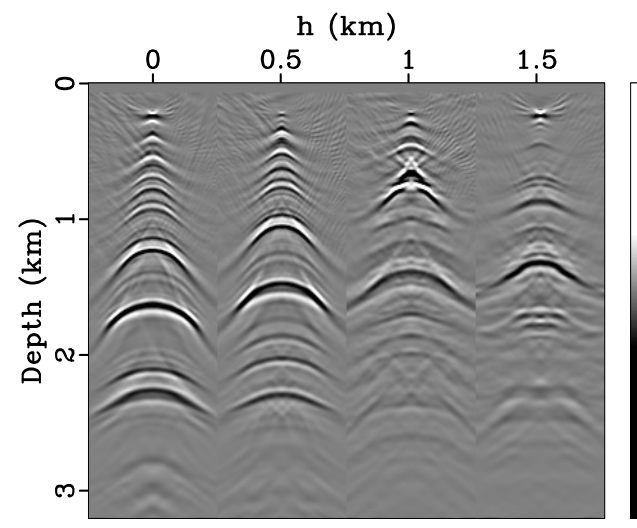

(c)

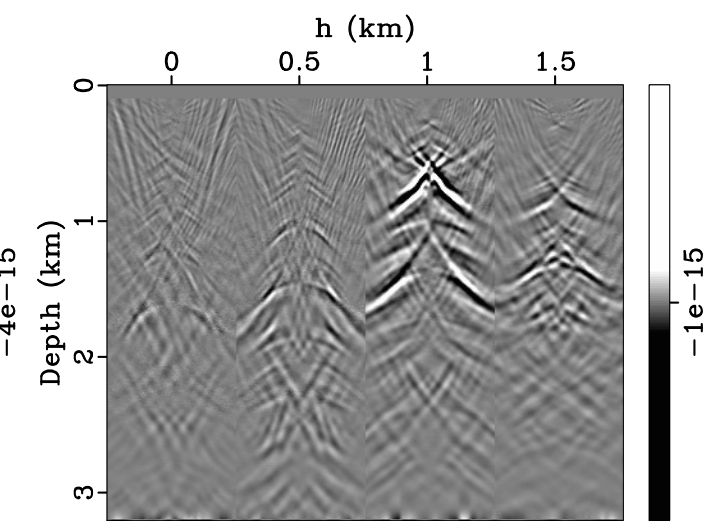

(b)

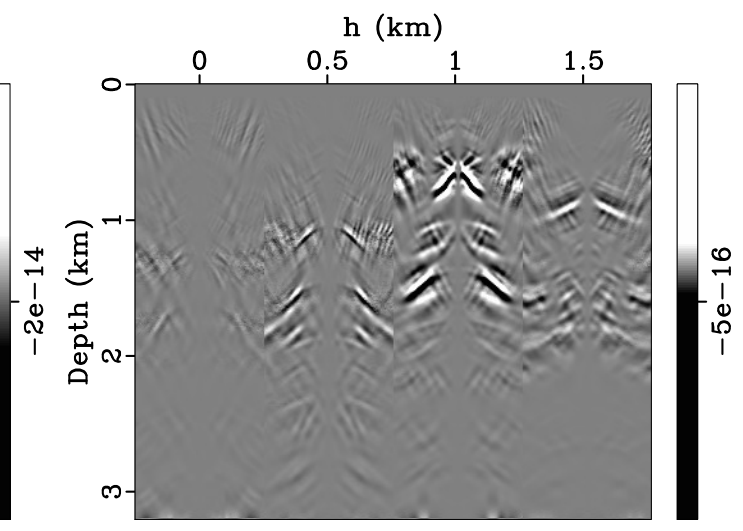

(d)

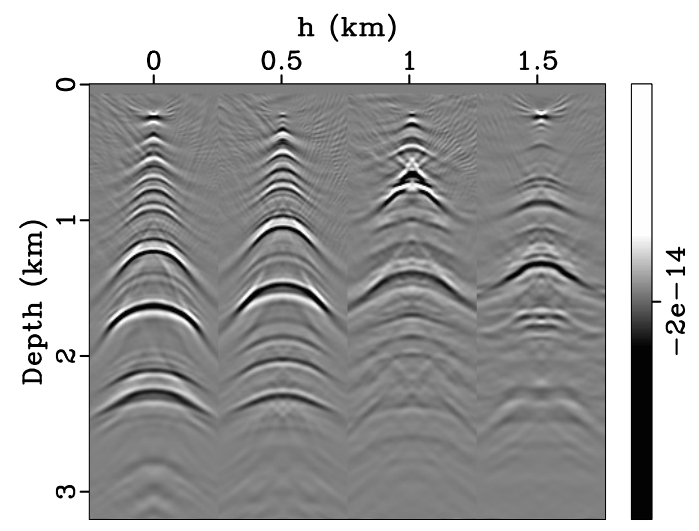

(e)

Figure 10: CIGs for the low velocity taken at $x=2,3,4.5$ and $6 \mathrm{~km}$ for (a) $D_{z}$ and (b) $D_{h_{z}}$ applied to the images and after multiplying them with the proper velocity weights in (c) and (d), respectively. (e) is the full $D_{p}$ operator. 

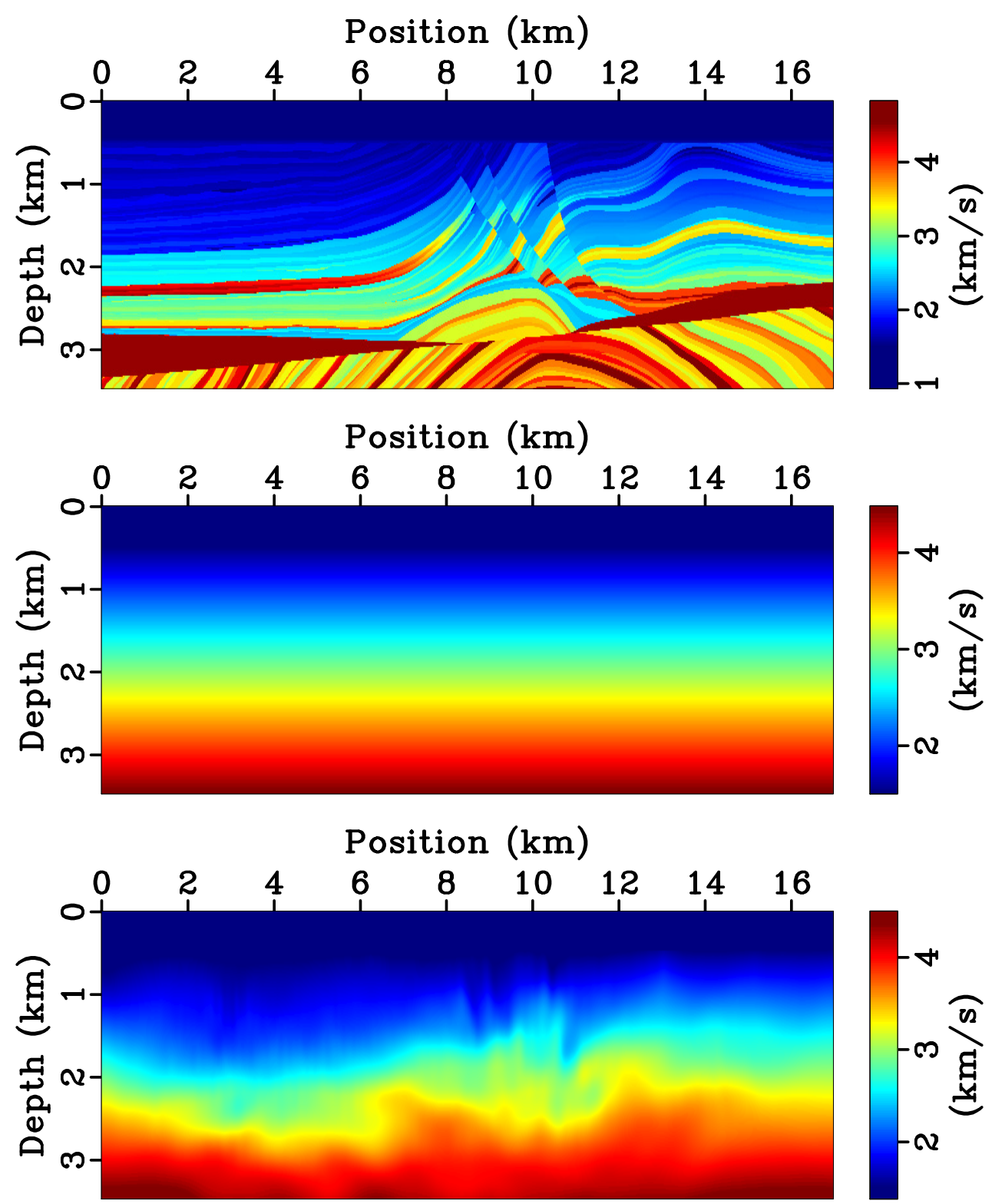

Figure 11: (a) is Marmousi (II) true model, (b) is the initial model and (c) the inverted model. 


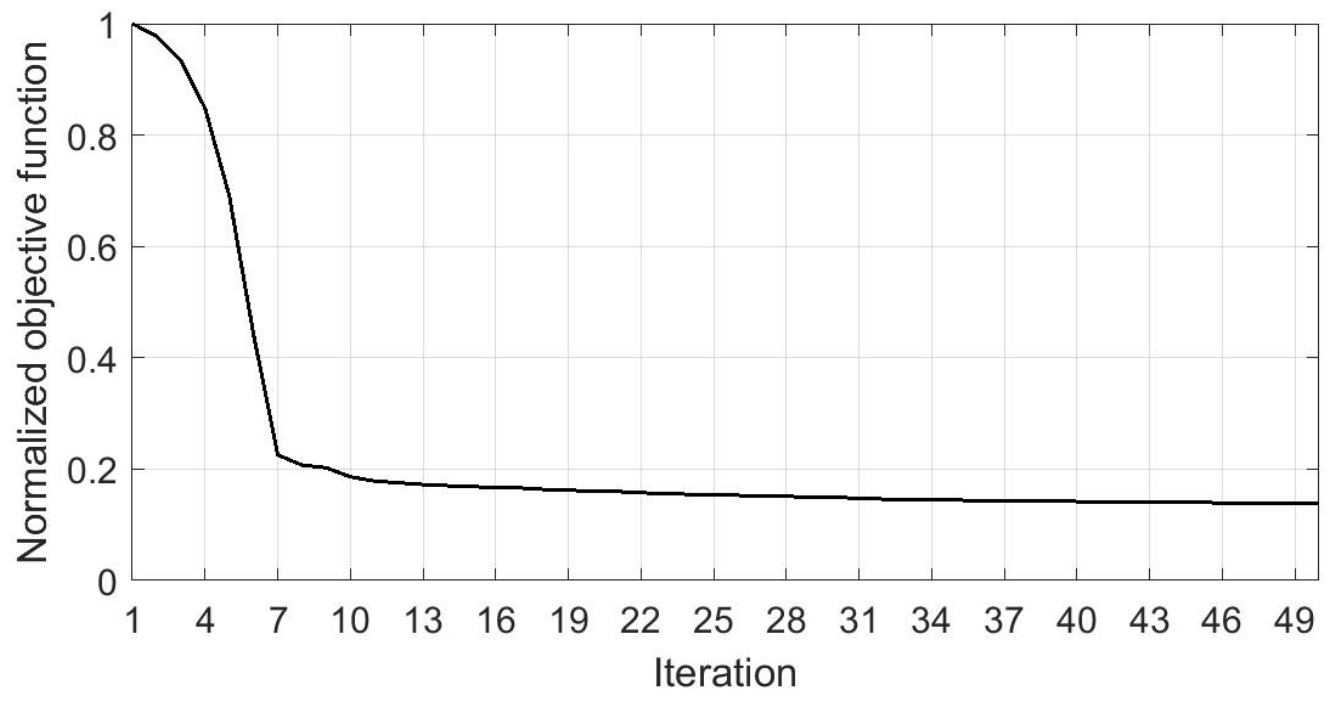

Figure 12: The convergence curve of the normalized objective function. 

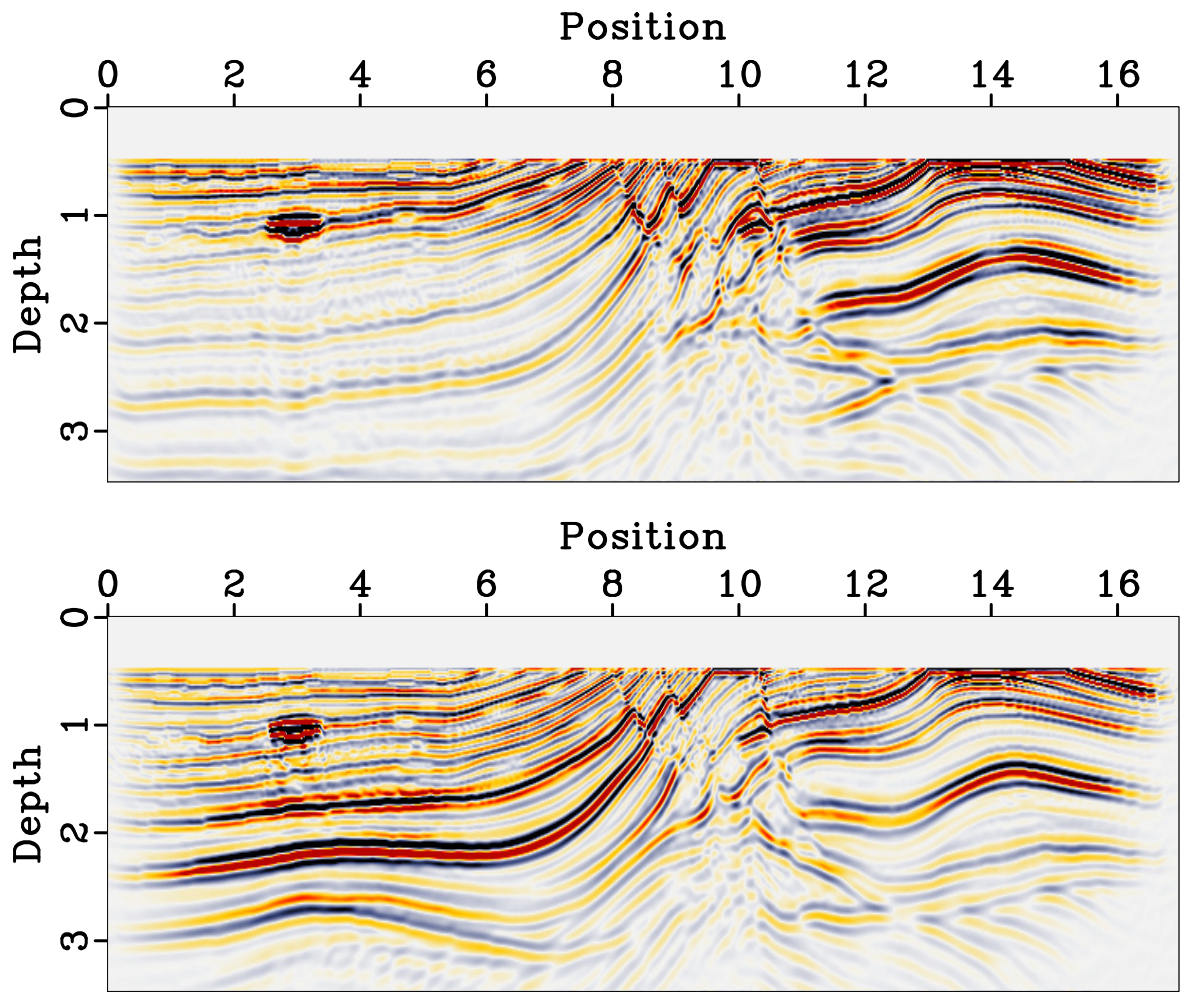

Figure 13: (a) is initial image and (b) the final image. 


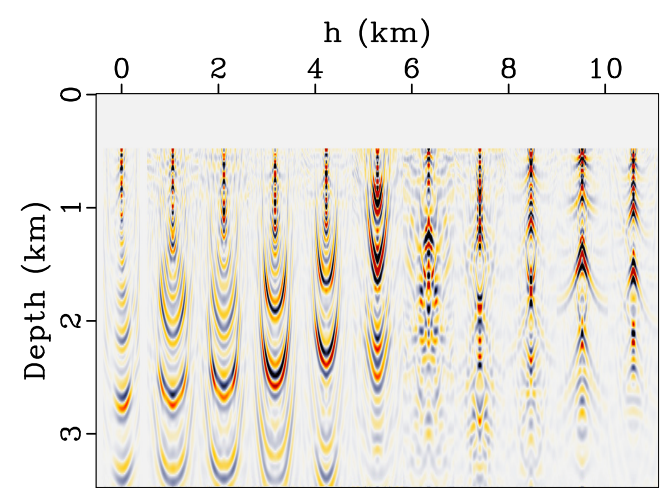

(a)

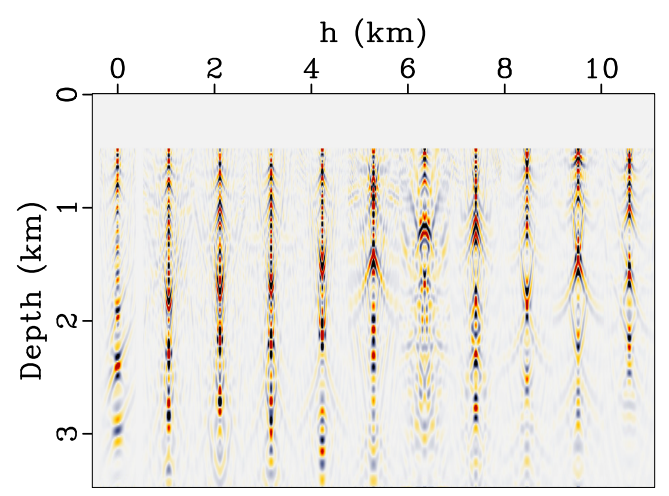

(b)

Figure 14: (a) is initial ODCIG and (b) is the final ODCIG. They are computed from $\mathrm{x}=0.75 \mathrm{~km}$ to $\mathrm{x}=15.75 \mathrm{~km}$ spaced by $1.5 \mathrm{~km}$ with maximum offset of $0.5 \mathrm{~km}$ 


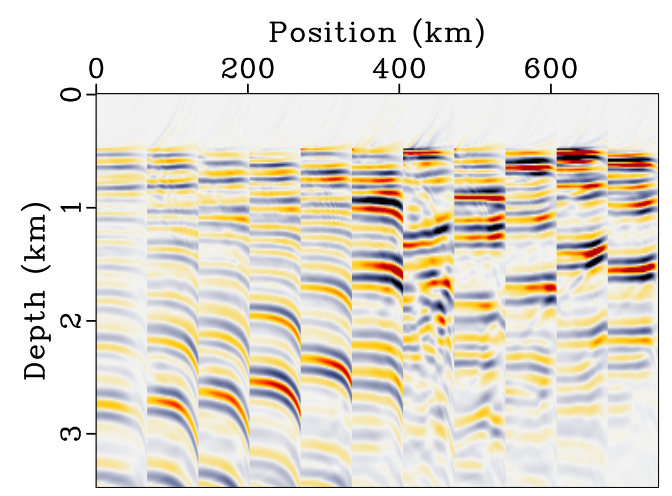

(a)

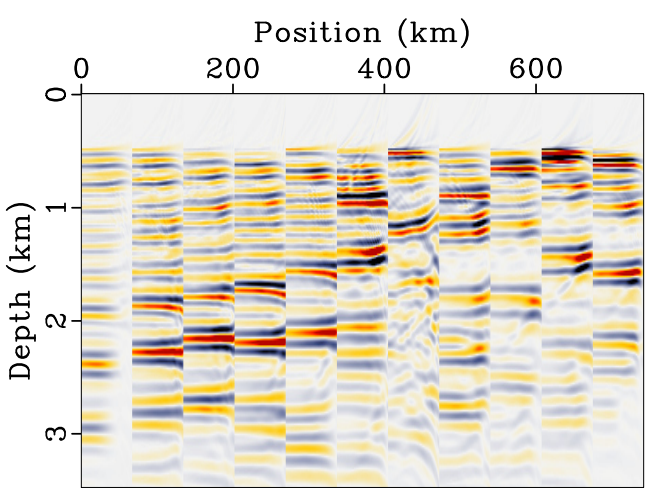

(b)

Figure 15: (a) is initial ADCIG and (b) is the final ADCIG obtained by converting the gathers in Figure 14 with a maximum incident angle of 67.5 degree. 


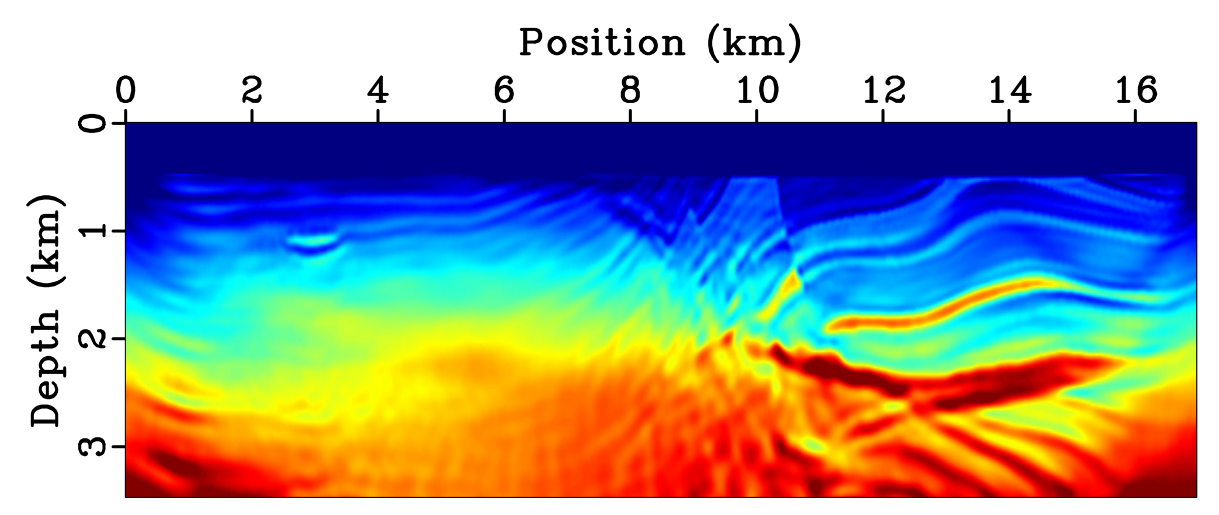

(a)

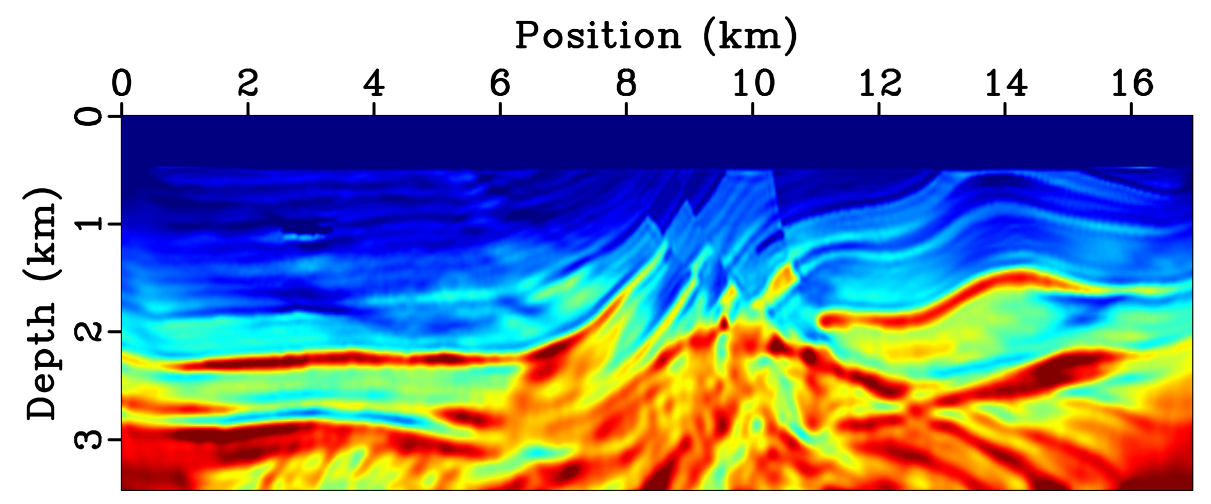

(b)

Figure 16: FWI results staring from the initial models (a) linearly increasing $v(z)$ model given in Figure 11(b), and (b) the IVA result shown in Figure 11(c) 